\title{
Clinical application of transanal ileal tube placement using $\mathrm{X}$-ray monitoring
}

\author{
DECHUN LI ${ }^{1}$, HONGTAO DU ${ }^{1}$, GUOQING SHAO ${ }^{1}$, YUANSHUN XU ${ }^{1}$, RUIHONG LI $^{2}$ and QINGZHONG TIAN ${ }^{3}$ \\ Departments of ${ }^{1}$ Radiology, ${ }^{2}$ Gastroenterology and ${ }^{3}$ General Surgery, \\ Xuzhou Central Hospital, Xuzhou, Jiangsu 221009, P.R. China
}

Received August 19, 2015; Accepted October 29, 2015

DOI: $10.3892 / \mathrm{ol} .2016 .5443$

\begin{abstract}
Colorectal cancer, which includes colon and rectal cancer, is a common digestive tract tumor. Although surgery is the primary form of treatment, there are a number of drawbacks, including patients experiencing considerable pain and high cost. The present study was undertaken to examine the clinical value of transanal ileal tube placement under X-ray monitoring. Thirty-six cases of left colon obstruction presenting to our hospital between July 2011 and February 2014, underwent transanal ileal tube placement using a single-curve catheter guided by a guidewire under $\mathrm{X}$-ray monitoring. An ileal tube was successfully inserted into 32 patients. Clinical symptoms were alleviated effectively within $48 \mathrm{~h}$. Indwelling catheter decompression time was 4-9 days with an average of 5.61 days. In two cases, the colon guidewire perforated into the abdominal cavity. Repeated exploration resistance of the guidewire and catheter indicated stenosis at this position owing to obstruction. In conclusion, transanal placement of the ileal tube through X-ray monitoring is capable of effectively alleviating the symptoms of ileus. Thus, this constitutes a safe, effective, and economical method that is acceptable to patients.
\end{abstract}

\section{Introduction}

Colorectal cancer, which includes colon and rectal cancer, is a common digestive tract tumor. In recent years, there is an upward trend in the incidence of colorectal cancer in China (1). The incidence of colorectal cancer in China is ranked fourth in men and third in women (2). Acute left-sided mechanical intestinal obstruction needs to be addressed. Traditional surgical treatment is utilized for gastrointestinal fistula, followed by staging tumor resection (3). Tumor resection is primarily used to treat patients. Although it is generally

Correspondence to: Dr Ruihong Li, Department of Gastroenterology, Xuzhou Central Hospital, 199 Jiefang North Road, Xuzhou, Jiangsu 221009, P.R. China

E-mail: liruihong7878@sina.com

Key words: X-ray, ileus tube, guidewire, intestinal obstruction, colon considered successful, there are disadvantages to consider, including the length of course of treament, the considerable pain experienced by patients and high cost. Middle-aged and elderly patients often experience a high percentage of water and electrolyte disorders, reulting in an increased risk of emergency surgery. There is also a high incidence of postoperative complications, such as anastomotic fistula (4).

Use of the ileal tube for bowel decompression has become increasingly common (5). The decompression tube was inserted into the proximal colon of obstruction point through anus to effectively alleviate and eliminate any obstruction in patients with symptoms, thereby avoiding emergency surgery. It also allows for intestinal cleaning, relieves swelling (edema) of the intestinal wall, creating conditions for tumor I stage radical excision anastomosis. Left colon obstruction may be treated by placing an ileal tube during colonoscopy and having patients undergo a single-stage anastomosis following colonic decompression and lavage, which reduces experience of pain (6-8). This process can cause secondary pain during treatment because of the ileal tube placement. Transanal placement of the ileal tube to treat left colon obstruction through X-ray monitoring has been widely used in Xuzhou Central Hospital since 2011. Patients readily accept this procedure as it is virtually painless, has a high success rate, and fewer complications.

The present study was undertaken to examine the clinical value of transanal ileal tube placement under X-ray monitoring.

\section{Patients and methods}

Patients. Thirty-six patients were diagnosed with left colon cancer obstruction in Xuzhou Central Hospital between July 2014 and February 2011. Of the 36 cases, 21 were men, and 15 were women, with an age range of 45-93 years and a median age of 73.5 years. The course of the disease was 1-7 days with an average of 3 days. All 36 patients were diagnosed with left-sided malignant tumor and obstruction through X-ray and computed tomography, including 8 cases of left-sided intestinal obstruction, 14 cases of descending colon obstruction, 10 cases of sigmoid colon obstruction and 4 cases of upper rectal obstruction. Patients had a different degree of abdominal distension and pain prior to treatment. Any patients with intestinal strangulation were excluded from the study. 


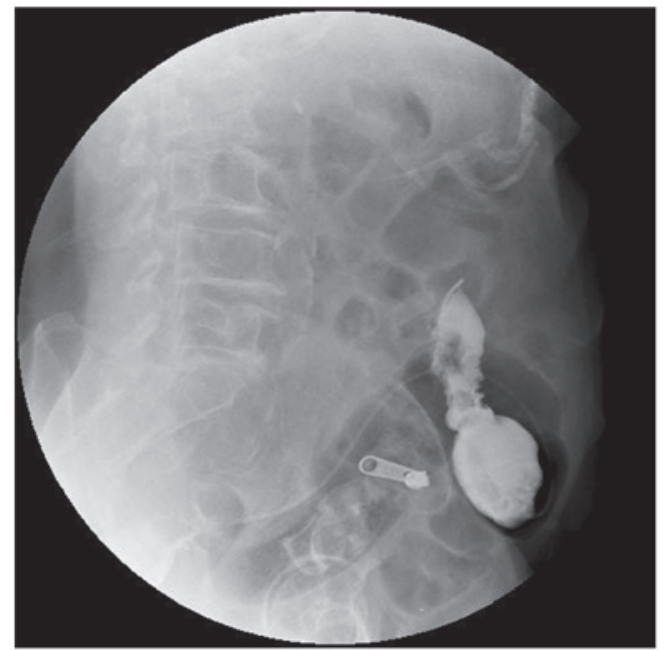

Figure 1. The guidewire and catheter cannot pass. Injected contrast agent shows that the contrast agent is blocked and cannot enter.

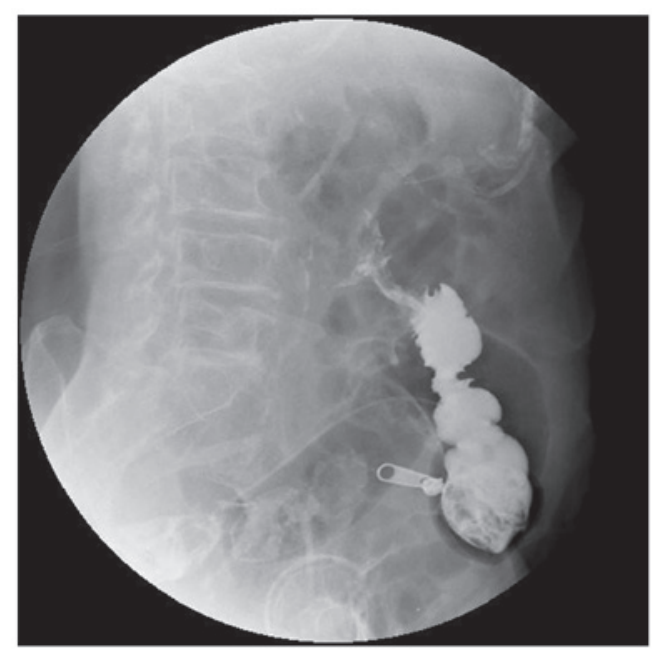

Figure 2. Transcatheter pressure injection of contrast agent shows partial stenosis of the intestinal lumen with a small amount of contrast agent entering.

Instruments. The Philips Tele-Diagnost digital gastrointestinal machine (Philips, The Netherlands) was used to produce $\mathrm{X}$-ray and guide the technical operation. The $(0.97 \mathrm{~mm})$ $2.6 \mathrm{~m}$ long guidewire was purchased from Terumo (China) Holding Corp. (Tokyo, Japan). The $4.2 \mathrm{~m}$ long intestinal guidewire (MTN-QF medical non-vascular guidewire) was purchased from Micro-Tech Co., Ltd. (Nanjing, China). The $5 \mathrm{~F}$ single-curve catheter (1.25 m long) was purchased from Cordis Corp. (Miami Lakes, FL, USA) and intestinal lavage syringes $(100 \mathrm{ml})$ were purchased from Shandong Weigao Group Medical Polymed Co., Ltd. (Weihai, China).

Methods. Patients underwent conventional abdominal vertical perspective to observe the obstruction site. The patient lay in the left lateral recumbent position, and the loach guidewire and $5 \mathrm{~F}$ single-curve catheter were inserted through the rectum and gently guided via X-ray monitoring. If the guidewire did not perforate, a single bend duct and iohexol were used to observe whether any contrast agent was diffused. If any contrast agent was detected, a different angle was used to follow the path

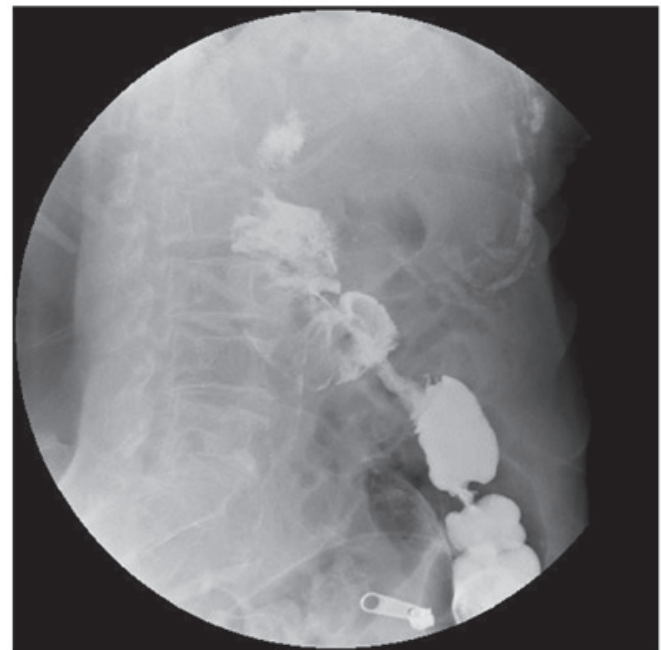

Figure 3. The guidewire passes through the narrow section along with the contrast agent.

of further insertion of the guidewire catheter. However, when no difference was observed in the vessels, repeated exploration using a combination of the guidewire and catheter was performed. The loach guidewire was withdrawn following perforation of the obstruction site. The contrast agent was injected by transcatheter to confirm that the catheter tip was located in the dilated colon. The site and length of obstruction were observed at the same time. The hard guidewire was then inserted, and the single-curve catheter was withdrawn. A dilatation catheter was inserted together with the intestinal guidewire for pre-expansion.

An ileal decompression tube coated with paraffin oil on the front was inserted along with the hard guidewire. When the balloon of the ileal decompression tube encountered the stenosis as confirmed by X-ray, the tube was inserted for a further $10 \mathrm{~cm}$ to prevent necrosis of the bowel wall regions caused by stenosis compression following infusion of water into the balloon. A total of $30 \mathrm{ml}$ in sterile distilled water was injected into the balloon to prevent prolapse of the intestinal obstruction decompression catheter. The Y-type joint was connected, and the contrast agent was again injected to confirm that the decompression tube was located in the colon (Figs. 1-5). The medical adhesive tape was used to fasten the rectum catheter postoperatively. Continuous suction was applied following the procedure and intermittent suction was applied on the second day. At the same time, full colon irrigation with warm water (500 ml/times) was applied 4-6 times a day (4). Abdominal pain, bloating, and other symptoms were carefully observed postoperatively. Properties of the drainage fluid were observed to confirm the drainage catheter patency.

\section{Results}

Thirty-two patients underwent successful transanal ileal decompression tube under X-ray monitoring. Thirty-one patients underwent decompression, intestinal lavage drainage and stage 1 intestinal resection and anastomosis. No cases of anastomotic leakage or death due to surgery were identified. However, incision infection was evident in one patient. One 


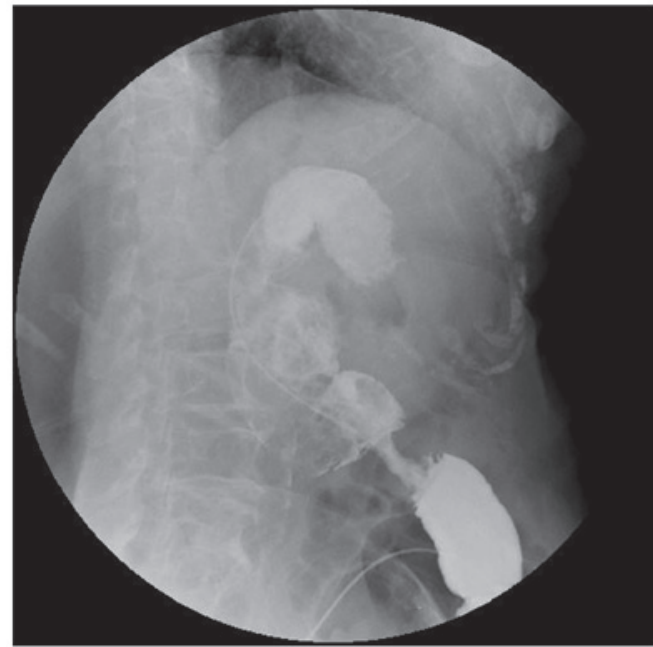

Figure 4. Decompression tube is inserted followed by the guidewire.

patient preferred non-surgical therapy (stenting) subsequent to the lifting of intestinal obstruction symptoms due to age.

Decompression time of catheter indwelling in 31 patients was 4-9 days (median 5 days, average 5.61 days). The catheter drainage observed after $24 \mathrm{~h}$ was yellow, brown sticky stool, mixed with a substantial amount of gas. Waste volume was 2,000-4,000 $\mathrm{ml}$ with an average of 2,800 $\mathrm{ml}$. Abdominal pain, and abdominal distension symptoms of all the patients were apparently alleviated. Colonic expansion was significantly improved (Fig. 6).

The guidewire perforated the bowel in two patients during exploration of the obstruction. Contrast agent diffusion into the abdominal cavity was evident after injection with the contrast agent. The catheter and guidewire were subsequently withdrawn and patients immediately underwent surgery. The repeated exploration guidewire did not perforate the obstruction site in two patients and tube indwelling was abandoned.

\section{Discussion}

Colonic obstruction is a serious complication of colorectal cancer indicating poor prognosis in patients. The primary option for colorectal cancer patients is surgery. For right-sided colonic cancer obstruction or chronic partial obstruction, stage 1 whole resection and anastomosis is preferred following sufficient preoperative bowel preparation $(9,10)$.

Regarding left-sided colon cancer, stage 1 complete resection and anastomosis potentially induce infection and anastomotic fistula due to prominent edema and dilatation of the proximal colon, poor blood supply, large amount of bacteria, and complexity of the bacteria (11). Tumor obstruction of the colon frequently occurs in elderly patients with further complications in multiple organs, compensatory dysfunction, immune dysfunction, reduced surgical tolerance, further surgical complications and high mortality rate (12). The traditional treatment approach is the Hartmann procedure (abdominal resection of rectal cancer, with a proximal colonic stoma and distal closure operation), followed by closure of the stoma in selective surgery $(3,9)$. However, this procedure involves a long period of recuperation time, significant pain,

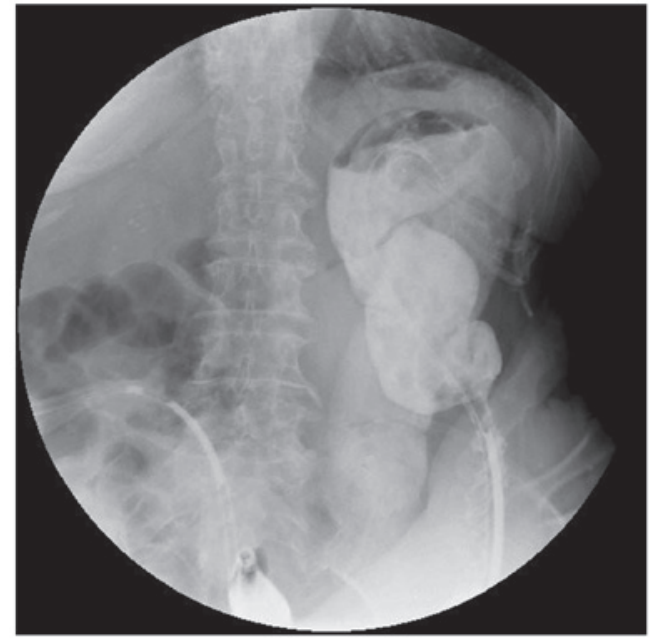

Figure 5. Injection of contrast agent through decompression tube in the colon and dilatation of the colon.

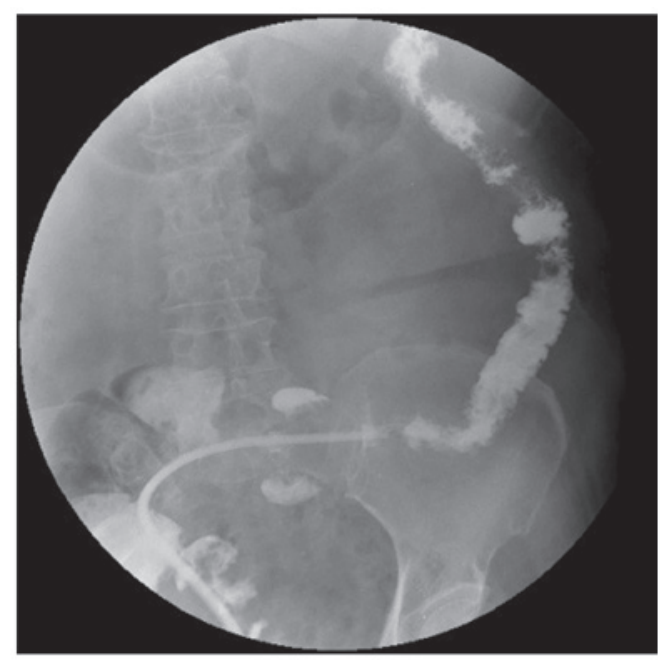

Figure 6. Improvement of the dilatation of the colon after decompression and lavage.

and high cost. In addition, patient quality of life is inevitably affected owing to the existence of the stoma. Stage 1 anastomosis can be performed when the bowel is prepared preoperatively. The ileus tube may be guided by an endoscope to provide lavage and obtain good results $(6,13)$. However, the endoscopic catheter requires preparation including enema bowel cleaning prior to insertion, as well as gas injection during colonoscopy which causes considerable pain (14). Consequently, this procedure is not preferred by patients. Transanal ileal tube placement under X-ray monitoring is more readily accepted by patients due to a lower amount of pain, high success rate, and observation of the direction of the catheter and the guidewire at any point.

In the present study, the path of the guidewire during exploration was different to that in the normal human colon. The contrast agent was injected through a single-curve catheter. If perforation of the catheter was confirmed, colonic investigation continued until the expanded colon was reached. In two cases, contrast agent diffusion following injection confirmed that the catheter had penetrated the abdominal cavity. The 
catheter and guidewire were withdrawn in a timely manner causing no visible peritonitis or other serious consequences.

Repeated exploration resistance of the guidewire and catheter indicates stenosis. Transcatheter injection of the contrast agent was used to determine whether the contrast agent was diffused, which would allow for insertion of the guidewire or catheter. No contrast agent diffusion indicated a short stenosis time and that the site of stenosis was not infiltrated or fused completely, but had a small gap in the majority of cases. The contrast agent was used by pressure injection to observe contrast agent infiltration. The majority of patients exhibited a small amount of contrast agent penetration. If contrast agent infiltration was successful, exploration involved guidewire combined with catheter, whereas if there was no contrast agent infiltration, careful inspection using guidewire and catheter individually was required. The guidewire guided the catheter in the exploration process. The contrast agent was injected in a timely manner every $2-3 \mathrm{~cm}$, to ensure that the front section of the catheter is in the middle of the colon. At the same time, the site and length of the obstruction were observed.

When the ileus tube was inserted, the catheter tip was coated with paraffin oil to facilitate its insertion. The catheter was inserted a further $10 \mathrm{~cm}$ after the decompression catheter balloon across the stenosis to prevent necrosis of the bowel wall caused by the water injection to the balloon. Since stools in the colon tend to be thick in general, leading to a fecal mass in some cases, the catheter must remain unobstructed. If blockage occurs, the timely use of an intestinal lavage syringe dredge is necessary. The dredging method involves the infusion of $500 \mathrm{ml}$ warm saline water into the intestine and is then suspended for $30 \mathrm{~min}$ after drainage. Warmed saline water was used for dredging the following day and retained for approximately $30 \mathrm{~min}$ to soften the stools. Attention was paid to water-electrolyte and acid-base balance in patients subsequent to insertion of the catheter.

In summary, transanal placement of the ileus tube under $\mathrm{X}$-ray monitoring effectively alleviates left-sided colonic obstruction symptoms following preoperative preparation. Thus, the present study has identified a safe, effective, economical treatment method that may be utilized for colon cancer and readily accepted by patients.

\section{References}

1. Chen W, Zheng R, Zeng H, Zhang S and He J: Annual report on status of cancer in China, 2011. Chin J Cancer Res 27: 2-12, 2015.

2. Jie $\mathrm{H}$ and Wanqing C: China tumor registration report. Military Medical Science Press, Beijing, pp31-56, 2012.

3. Breitenstein S, Rickenbacher A, Berdajs D, Puhan M, Clavien PA and Demartines N: Systematic evaluation of surgical strategies for acute malignant left-sided colonic obstruction. Br J Surg 94: 1451-1460, 2007.

4. Pavlidis TE, Marakis G, Ballas K, Rafailidis S, Psarras K, Pissas D, Papanicolaou K and Sakantamis A: Safety of bowel resection for colorectal surgical emergency in the elderly. Colorectal Dis 8: 657-662, 2006.

5. Nozoe T and Matsumata T: Usefulness of preoperative colonic lavage using transanal ileus tube for obstructing carcinoma of left colon: device to perform one-stage operation safely. J Clin Gastroenterol 31: 156-158, 2000.

6. Dekovich AA: Endoscopic treatment of colonic obstruction. Curr Opin Gastroenterol 25: 50-54, 2009.

7. Fischer A, Schrag HJ, Goos M, Obermaier R, Hopt UT and Baier PK: Transanal endoscopic tube decompression of acute colonic obstruction: experience with 51 cases. Surg Endosc 22: 683-688, 2008

8. Araki Y, Isomoto H, Matsumoto A, Kaibara A, Yasunaga M, Hayashi K, Yatsugi H and Yamauchi K: Endoscopic decompression procedure in acute obstructing colorectal cancer. Endoscopy 32: 641-643, 2000.

9. Villar JM, Martinez AP, Villegas MT, Muffak K, Mansilla A, Garrote D and Ferron JA: Surgical options for malignant left-sided colonic obstruction. Surg Today 35: 275-281, 2005.

10. Ohnita K, Shikuwa S, Isomoto H, Yamaguchi N, Okamoto K, Nishiyama H, Fukuda E, Nakamura T, Mizuta Y and Kohno S: A new thin endoscopic method of transanal drainage tube insertion for acute colonic obstruction due to colorectal cancer. Dig Endosc 21: 252-254, 2009.

11. Leong QM, Aung MO, Ho CK and Sim R: Emergency colorectal resections in Asian octogenarians: Factors impacting surgical outcome. Surg Today 39: 575-579, 2009.

12. Merkel S, Meyer C, Papadopoulos T, Meyer T and Hohenberger W: Urgent surgery in colon carcinoma. Zentralbl Chir 132: 16-25, 2007 (In German).

13. Morino M, Bertello A, Garbarini A, Rozzio G and Repici A: Malignant colonic obstruction managed by endoscopic stent decompression followed by laparoscopic resections. Surg Endosc 16: 1483-1487, 2002.

14. Keränen I1, Lepistö A, Udd M, Halttunen J and Kylänpää L: Stenting for malignant colorectal obstruction: a single-center experience with 101 patients. Surg Endosc 26: 423-430, 2012. 